Sánchez Ordóñez Maria J.

Odontóloga Especialista en Endodoncia,

Universidad de Buenos Aires, Argentina,

Especialista en Implantología Oral, Pontificia Universidad Católica

Argentina Santa María de los Buenos Aires.

Docente Titular Carrera de Odontología de la Universidad Católica

de Cuenca, Sede Azogues, Ecuador

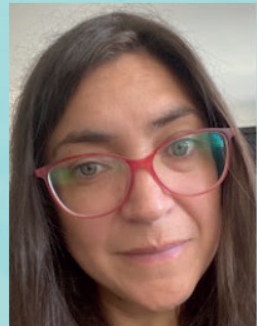

Medina Sotomayor Priscilla

Odontóloga Especialista en Dentistica, Faculdade Uninga, Brasil Master en Ciencias Odontológicas, Universitat de Valencia, España Doctorado en Odontología (PhD.), Universitat de Valencia, España Docente Titular Carrera de Odontología de la Universidad Católica de Cuenca,

Sede Azogues, Ecuador

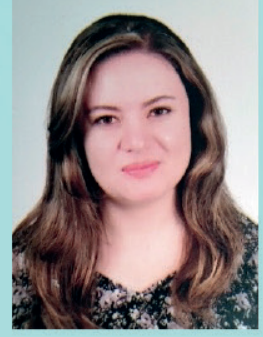

Montesinos Rivera Vanessa

Odontóloga Especialista en Endodoncia, Universidad de Cuenca, Ecuador

Diplomado Superior en Odontología Restauradora y Estética, Universidad de Cuenca, Ecuador Diploma Superior en Didáctica Universitaria en Ciencias de la Salud Universidad de Cuenca, Ecuador

Diploma superior en Fundamentos de Técnicas de Endodoncia

Universidad de Cuenca, Ecuador,

Docente Titular Carrera de Odontología de la Universidad Católica de Cuenca,

Sede Azogues, Ecuador

\title{
ESTUDIO IN VITRO
}

DE LOS ERRORES DE

PROCEDIMIENTO EN LOS

TRATAMIENTOS DE

CONDUCTO REALIZADOS

POR ESTUDIANTES DE

PREGRADO: UNA EVALUACIÓN

RADIOGRÁFICA

POSTOPERATORIA

I N VITRO STUDY OF

PROCEDURAL ERRORS

I N ROOT CANAL TREATMENT

B Y UNDERGRADUATE DENTAL

STUDENTS: A POSTOPERATIVE

RADIOGRAPHIC EVALUATION 


\section{RES U M E N}

Es importante mejorar los programas de pregrado, donde es posible reconocer las razones que afectan el éxito de los tratamientos dentales. Una práctica preprofesional correcta debe estar vinculada a la calidad del tratamiento ofrecido en la práctica dental general. Objetivo: analizar radiográficamente los errores de procedimiento cometidos por estudiantes de pregrado de odontología en la cátedra de endodoncia durante la práctica pre profesional para determinar la calidad del tratamiento. Material y métodos: se analizó la radiografía periapical postoperatoria en una muestra de 1226 raíces con tratamiento endodóntico. Se evaluó la preparación del conducto, obturación y grado de condensación del tratamiento. Resultados: se evidenció una correcta preparación en el $96 \%$ de casos, pero en el $50 \%$ de los tratamientos se encontró una sub obturación y mala condensación. Conclusiones: los errores de procedimiento en tratamientos endodónticos de los estudiantes de pregrado reflejan una calidad técnica de obturación radicular baja, lo que puede revelar que la educación endodóntica tiene un rendimiento limitado a nivel de pregrado.

Palabras clave: Terapia de conductos, errores de procedimiento, calidad de procedimiento, estudiantes dentales, educación dental, evaluación radiográfica.

\section{ABSTRACT}

It is important to improve undergraduate programs, where it is possible to recognize the reasons that affect the success of dental treatments. A correct pre-professional practice must be linked to the quality of the treatment offered in general dental practice. Objective: the purpose of this study was to analyze radiographically the procedural mistakes made by dentistry students in the subject of endodontics during pre-professional practice. Materials and methods: postoperative periapical radiography was analyzedin a sample of 1226 roots with endodontic treatment. The canal preparation, obturation and degree of condensation were evaluated. Results: proper preparation was evident in $96 \%$ of cases, but in 50\% of the treatments there was a low speed-obturation and poor condensation. Conclusions: the technical quality of root obturation performed by students is low, which may reveal that endodontic education has limited performance at the undergraduate level.

Key Words: root canal therapy, procedural errors, procedural quality, dental students, dental education, radiographic evaluation. 


\section{INTRODUCCIÓN}

La relativa simplicidad y uniformidad macroscópica de las superficies externas de las raíces dentales a menudo enmascaran la complejidad de sus configuraciones internas dificultando la obturación tridimensional del sistema de conductos radiculares. La evaluación radiográfica postoperatoria es un método común para asegurar la calidad del tratamiento endodóntico y determinar su pronóstico. El conocimiento de la anatomía apical y la correcta interpretación radiográfica durante las diferentes etapas del tratamiento son esenciales para lograr el éxito en endodoncia. (1-6).

El proceso de limpieza y conformación del conducto no es un objetivo fácil de lograr, especialmente en conductos curvos. El área más difícil de limpiar y mantener es la zona apical; la formación de codos, transporte o zip apical, fractura de instrumentos y perforaciones son algunos de los accidentes que se pueden producir al preparar el conducto radicular y que conlleva al fracaso del tratamiento endodóntico (7). La calidad de la obturación final también puede provocar complicaciones post-tratamiento, debido a que si el material de obturación se encuentra entre los 0.5 a $2 \mathrm{~mm}$ del ápice radiográfico se puede considerar que los fracasos serán mínimos (8), mientras que cada milímetro sin trabajar incrementa el fallo del tratamiento en un 14\% (9).

Previos estudios reportan índices de éxito en los tratamientos endodónticos por encima del $90 \%$ cuando son realizados por especialistas $(10,11)$, sin embargo, este éxito disminuye al 40-65\% cuando es realizado por odontólogos generales, más aún si son realizados por estudiantes de pregrado (12). Esta disminución se puede atribuir a la insuficiencia de los programas educativos, relacionados a la calidad y tiempo que emplean los estudiantes a perfeccionar dichos tratamientos. Por esta razón, es importante mejorar los programas de pregrado, donde es posible reconocer las razones que afectan el éxito de estos tratamientos (5) mejorando la práctica pre profesional que estará ligada a la calidad del tratamiento ofrecido en la práctica dental general.

El objetivo del presente estudio fue analizar radiográficamente los errores de procedimiento cometidos por estudiantes de pregrado de la Carrera de Odontología de la Universidad de Buenos Aires en la cátedra de endodoncia durante su práctica pre profesional evaluando la preparación del conducto, obturación y condensación para determinar la calidad del tratamiento.

\section{MATERIAL Y MÉTODOS}

El presente estudio retrospectivo, transversal se realizó como trabajo de titulación para obtener el grado de especialista en endodoncia en el año 2017, siendo aprobado por el comité evaluador. Contó con una muestra total de 847 piezas dentales extraídas que incluían incisivos, caninos, premolares y molares ubicadas en tacos de compuesto de modelar para proporcionarle estabilidad. Las piezas dentales extraídas fueron obtenidas de las unidades de salud odontológica pública de la ciudad de Buenos Aires, Argentina.

Se analizaron un total de 1226 conductos que fueron tratados endodonticamente de manera manual durante las prácticas preprofesionales de la cátedra de endodoncia de la Universidad de Buenos Aires por los estudiantes de pregrado, se incluyeron todas las muestras con las que contaba el departamento de endodoncia de la universidad; siendo un estudio retrospectivo, no se pudo determinar las técnicas utilizadas para realizar el tratamiento endodóntico, esto incluye la apertura, irrigación, preparación o limas utilizadas, por esta razón solo se analizó radiográficamente el resultado final del tratamiento endodóntico mediante una radiografía postoperatoria.

Cuatro observadores especialistas en endodoncia 
realizaron el análisis de las radiografías postoperatorias; para la división de la muestra se utilizó una aleatorización simple. Se evaluó el tratamiento endodóntico de cada conducto radicular mediante la observación de la radiografía periapical postoperatoria sobre un negatoscopio (Abbimed), con una lupa de magnificación de 4x (T\&Z, modelo Tzm-1025).

Las variables analizadas fueron: errores cometidos durante la preparación del conducto, obturación y condensación $(5,13,14)$

\section{Preparación del conducto:}

Se evaluó radiográficamente la presencia o no de: - Hombro, escalón o Transporte Apical interno: pérdida de la longitud de trabajo que se produce cuando no se logra el acceso directo a la porción apical del conducto por utilizar limas de grandes calibres en conductos curvos. (Fig. 1a)

- Perforación Radicular: destrucción de la dentina y el cemento a nivel del tercio coronario, medio o apical del conducto radicular, como consecuencia del mal uso de instrumentos endodónticos durante la preparación. (Fig. 1b)

- Fractura del instrumento: desprendimiento de una porción del instrumento en el interior del conducto por utilizar instrumentos muy tensionados, precurvados exageradamente o forzados en el interior del conducto cuando no ha sido ampliado lo suficiente. (Fig. 1c)

- Bloqueo del conducto: Formación de un tapón de barro dentinario que se produce por la falta de irrigación del conducto durante la preparación biomecánica. (Fig. 1d)

- Transporte apical externo: deformación del trayecto apical del conducto radicular. Se produce cuando la instrumentación se lleva hasta el fin del conducto radicular o más allá de él y produce el desgarro con deformación del extremo apical. (Fig. 1e)

- Zip o reloj de arena: tallado en forma elíptica del foramen apical de un conducto curvo durante la preparación, cuando una lima se extiende a través del forman y lo ensancha en forma de cono invertido. (Fig. 1f)

\section{Calidad de la obturación:}

Se evaluó radiográficamente:

- Obturación ideal: el límite apical de la obturación coincide con el límite ideal de la preparación del conducto (0.5 a $2 \mathrm{~mm}$ del extremo anatómico) y está bien condensada. (Fig. 2a)

- Sobreobturación: sobrepasa el límite ideal de la preparación del conducto, pero está bien condensada. (Fig. 2b)

- Sobreextensión: sobrepasa el límite ideal de la preparación del conducto, pero está mal condensada. (Fig. 2c)

- Subobturación: no alcanza el límite ideal de la preparación del conducto o bien puede alcanzarlo, pero está mal condensado. (Fig. 2d)

- Corta: no alcanza el límite ideal de la preparación del conducto, pero está bien consensada.

\section{Grado de condensación de la obturación:}

Se evaluó radiográficamente:

- Bien condensada: buena adaptación del material a las paredes del conducto y ausencia de poros o burbujas en el interior del material de obturación. (Fig. 3a)

- Mal condensada: deficiente adaptación del material a las paredes del conducto y presencia de poros o burbujas en el interior del material de obturación. (Fig. 3b)

Los datos se recogieron e introdujeron en el programa Infostat versión 2013, se obtuvo una estadística descriptiva y se calcularon intervalos de confianza al 95\% (IC95) para las estimaciones de porcentajes mediante el método score de Wilson.

\section{RESULTADOS}

En relación con la preparación del conducto, el total de 1226 tratamientos de conductos analizados se encontró 1187 tratamientos con una correcta preparación (96.82\%), y el error más 
frecuente fue el transporte apical externo con 25 casos (2.04\%). No se encontraron perforaciones radiculares. (Tabla 1)

En relación a la calidad de obturación se registró que en 279 casos (23\%) se realizó una obturación ideal, en 169 casos (14\%) se observaron obturaciones cortas y en 658 casos (54\%) se registran subobturaciones. (Tabla 2)

En relación al grado de condensación de la obturación se evidenció en 735 casos (60\%) una mala condensación. (Tabla 3)

\section{DISCUSIÓN}

El presente estudio fue realizado para evaluar los errores de procedimiento cometidos por estudiantes de pregrado durante su práctica pre profesional, analizando la preparación del conducto, obturación y condensación en la radiografía periapical postoperatoria. Cuatro especialistas en endodoncia evaluaron las radiografías, sin embargo, ningún análisis de concordancia fue realizado, lo que debe ser considerado al analizar los resultados.

Se utilizó radiografías periapicales postoperatorias del tratamiento endodóntico debido a que son capaces de exhibir la preparación y la obturación final para su correcta evaluación, sin embargo, siendo muestras obtenidas de trabajos realizados previamente, fue imposible determinar las técnicas utilizadas para la apertura, irrigación, preparación y obturación, centrando el estudio únicamente en el resultado final del tratamiento odontológico que puede ser capaz de definir el pronóstico de este $(8,9)$.

Los criterios radiográficos para evaluar la calidad del tratamiento endodóntico se establecieron de acuerdo con las directrices europeas y estudios clínicos previos $(5,13,14)$.

Los errores durante la realización del tratamiento endodóntico se producen por la complejidad anatómica y grado de calcificación que presentan los conductos, aumentando el riesgo de fracaso del tratamiento (11).

El presente estudió determinó que el 96\% de tratamientos tuvieron una correcta preparación de conducto,lo que indica que los estudiantespueden ser capaces de enfrentar los desafíos inherentes que presenta la morfología dental disminuyendo errores que puedan cometer en la práctica clínica. Estudios previos encuentran resultados similares, con un $87 \%$ de casos con una correcta instrumentación, que indica que las destrezas adquiridas en el pregrado pueden mejorar el pronóstico del tratamiento en la práctica general $(15,16)$

El transporte apical externo es el error más frecuente en la preparación del conducto con un 2,3\%, siendo consistente con resultados encontrados en otros estudios $(16,17)$.Este error puede ser el origen de diferentes complicaciones operatorias, debido a que la curvatura que presenta el ápice radicular puede dificultar su preparación y sellado siendo determinantes en el pronóstico de éxito o fracaso del tratamiento de endondoncia, sin embargo, este error mejora con el uso de sistemas rotatorios (18).

El error de obturación más frecuente fue la sub obturación con un 54\%.Su principal característica llegar al límite ideal de la preparación del conducto, pero sin condensarlo de manera incorrecta (60\%). Esto puede deberse a que cada pieza dental posee una morfología diferente, siendo los caninos quienes presentan una longitud más larga haciéndolo menos susceptible de sobre extender la obturación. Sin embargo, existen estudios que indican que si la gutapercha se extiende por el ápice tiene un efecto de cicatrización mínimo que puede ayudar al pronóstico del tratamiento endodóntico, por el contrario, la falta de gutapercha puede provocar el fracaso del tratamiento debido a la multiplicación de las bacterias residuales (17, 1920). Siendo tópicos muy controversiales lo mas 
seguro es controlar estos errores con una correcta condensación de la obturación final (17, 19-25).

Los tratamientos endodónticos fueron realizados de manera manual, actualmente se utiliza aparatología rotatoria que facilita la instrumentación y obturación final, sin embargo, existen estudios que descartan esta variable como importante a la hora de predecir el éxito de un tratamiento, debido a que es la dificultad que presenta la morfología dentaria o el caso clínico es la que está asociada a fracasos en los tratamientos realizados por estudiantes de pregrado $(26,27)$. Se debe mejorar la capacitación brindada a los estudiantes de pregrado para aumentar las destrezas clínicas con las que van a trabajar en la práctica odontológica general (28).

\section{CONCLUSIÓN}

El presente estudio pudo determinar que los tratamientos endodónticos realizados por los estudiantes de pregrado de la Facultad de Odontología de la Universidad de Buenos Aires tienen una correcta preparación del conducto, sin embargo, la técnica de obturación presenta una alta prevalencia de mala condensación y subobturación de los conductos radiculares.

\section{ANEXOS Y TABLAS}

Tabla 1

Distribución de la frecuencia de los errores

de procedimiento en relación con la preparación

del conducto.

\begin{tabular}{|c|c|c|c|c|}
\hline & & $\mathbf{N}$ & $\%$ & $\mathrm{IC}_{95}$ \\
\hline \multirow{8}{*}{$\begin{array}{c}\text { ERRORES } \\
\text { DE } \\
\text { PROCEDIMIENTO }\end{array}$} & Fractura del instrumento & 7 & 0,57 & $0,28-1,17$ \\
\hline & Transporte apical interno & 25 & 2,04 & $1,38-2,99$ \\
\hline & Transporte apical externo & 4 & 0,33 & $0,13-0,84$ \\
\hline & Zip o reloj de arena & 2 & 0,16 & $0,04-0,59$ \\
\hline & Bloqueo del conducto & 1 & 0,08 & $0,01-0,46$ \\
\hline & Perforación radicular & 0 & 0,00 & 0,00 \\
\hline & Sin error & $1187^{*}$ & $96,82^{*}$ & $95,68-97,66$ \\
\hline & TOTAL & 1226 & 100,00 & \\
\hline
\end{tabular}


Tabla 2

Distribución de la frecuencia de los errores de procedimiento en relación con la calidad de obturación del sistema de conductos.

\begin{tabular}{|c|c|c|c|c|}
\hline & & $\mathbf{N}$ & $\%$ & $\mathrm{IC}_{95}$ \\
\hline \multirow{6}{*}{$\begin{array}{c}\text { CALIDAD } \\
\text { DE LA } \\
\text { OBTURACIÓN }\end{array}$} & Ideal & 279 & 23 & $20-25$ \\
\hline & Sobre extensión & 88 & 7 & $6-9$ \\
\hline & Sobre obturación & 32 & 2 & $2-4$ \\
\hline & Corta & 169 & 14 & $12-16$ \\
\hline & Sub obturación & $658 *$ & $54 *$ & $51-56$ \\
\hline & TOTAL & 1226 & 100,00 & \\
\hline
\end{tabular}

Tabla 3

Distribución de la frecuencia de los errores de procedimiento en relación con el grado de condensación de la obturación del sistema de conductos.

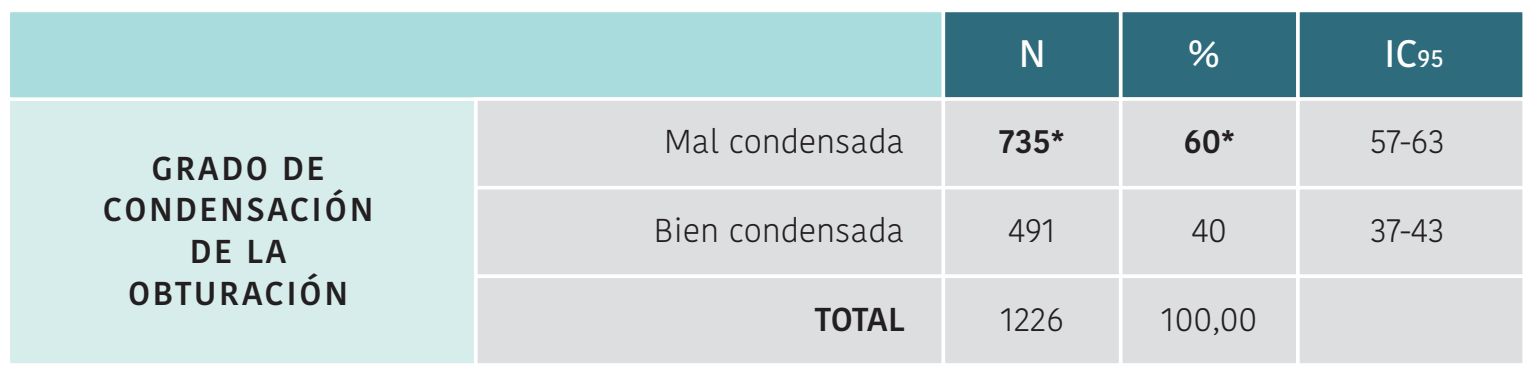

Figura 1

Radiografía post operatoria de los errores en la preparación del conducto. a) hombro, escalón o transporte apical interno, b) perforación radicular, c) fractura de instrumento, d) bloqueo de conducto, e) Transporte apical externo, f) Zip o reloj de arena.
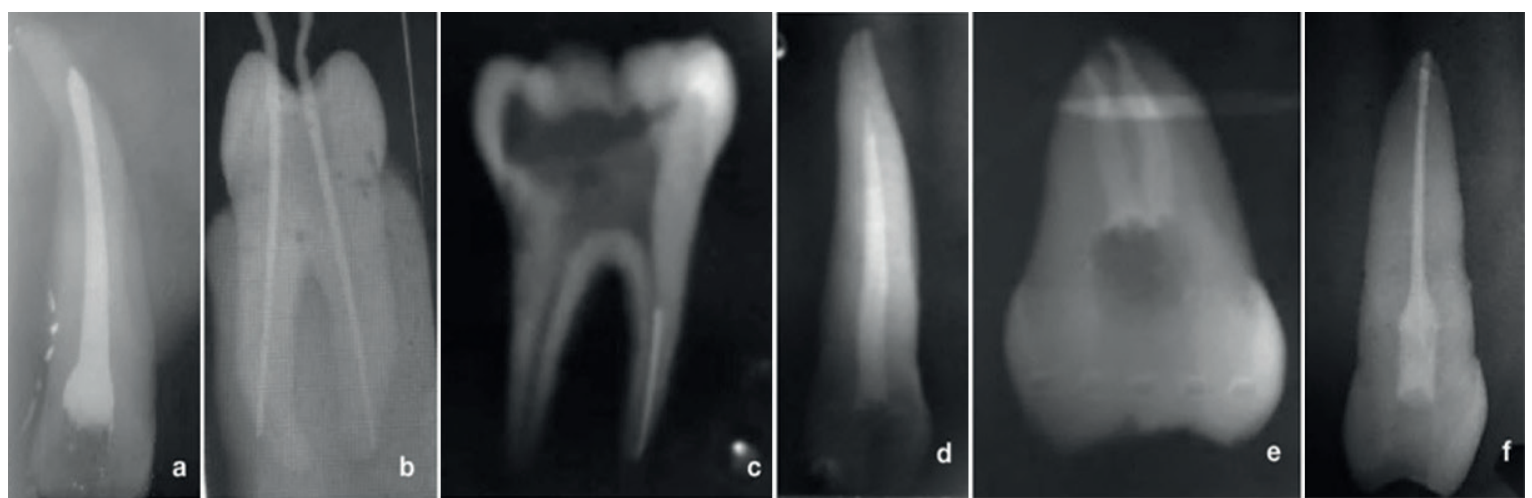
Figura 2

Radiografía post operatoria de la calidad de la obturación. a) obturación ideal, b) sobre obturación, c) sobre extensión, d) sub obturación, e) corta
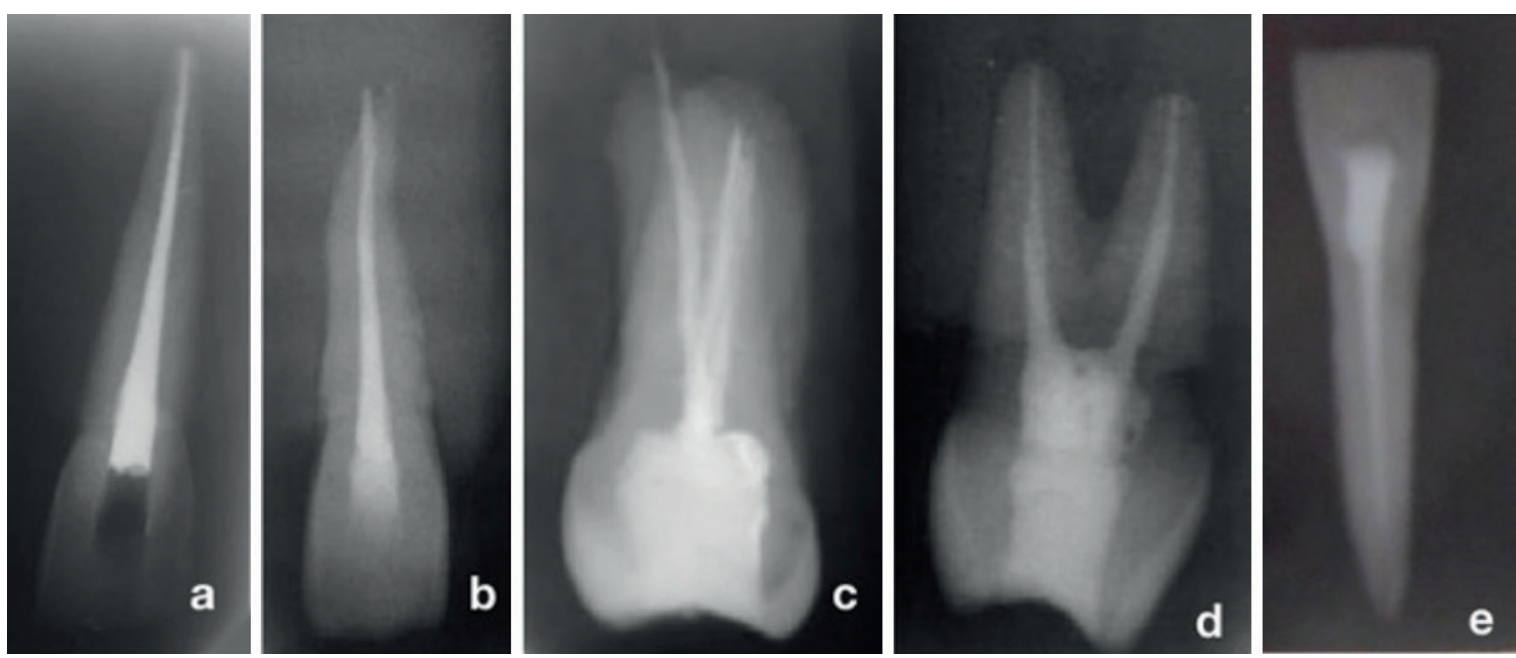

Figura 3

Radiografía post operatoria del grado de condensación de la obturación.

a) bien condensada, b) mal condensada

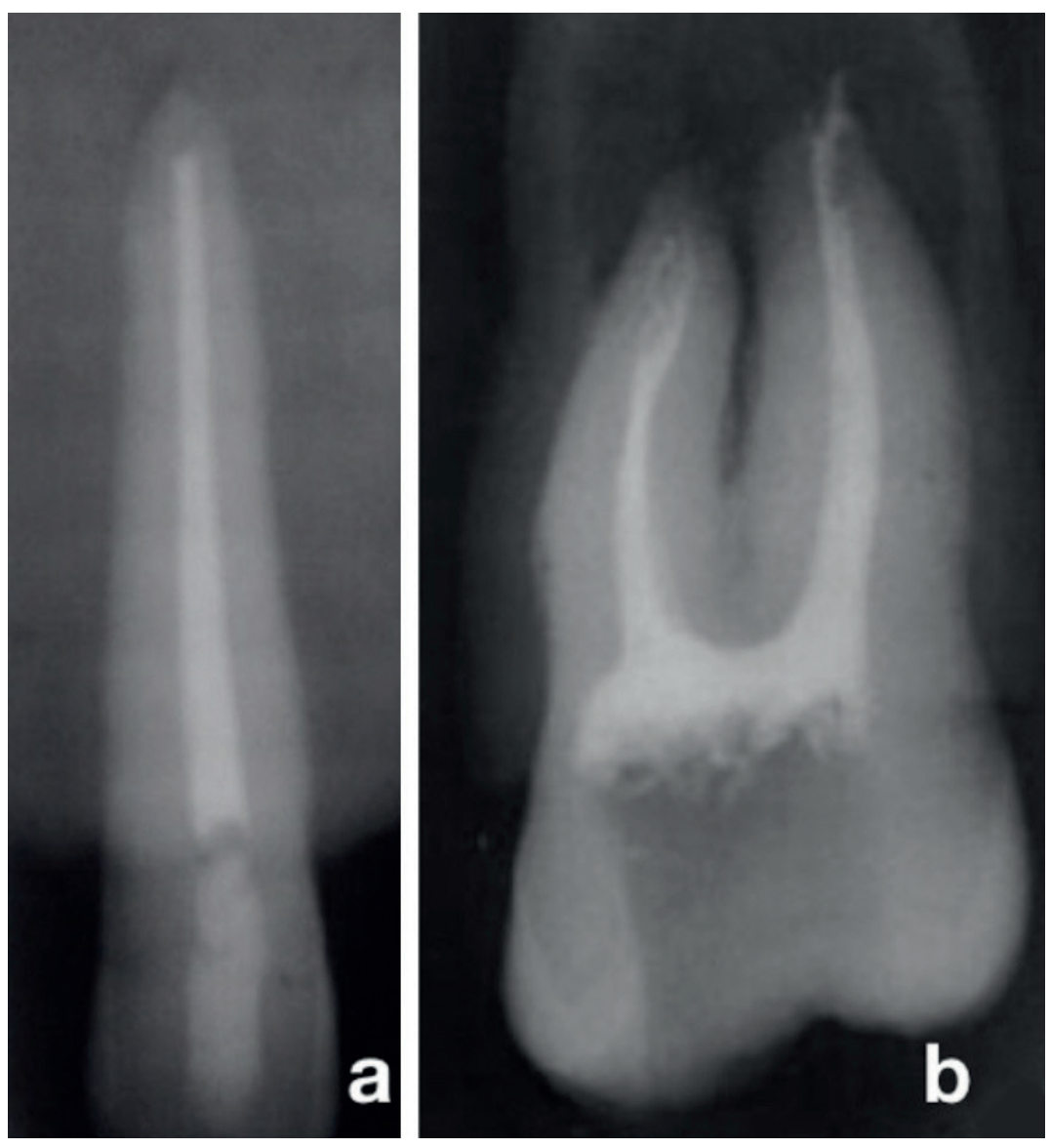




\section{B I B LIOGRAFÍA}

1. Hansrani V. Assessing root canal fillings on a radiograph-an overview. Br Dent J. 2015;219(10):481-483

2. Tsuneishi M, Yamamoto T, Yamanaka R, et al. Radiographic evaluation of periapical status and prevalence of endodontic treatment in an adult Japanese population. Oral Surg Oral Med Oral Pathol Oral Radiol Endod. 2015;100(5):631-635.

3. Khabbaz M, Protogerou E, Douka E. Radiographic quality of root fillings performed by undergraduate students. Int Endod J. 2010;43(6):499-508.

4. Moussa-Badran S, Roy B, Bessart du Parc A, et al. Technical quality of root fillings performed by dental students at the dental teaching centre in Reims, France. Int Endod J. 2008;41(8):679-84.

5. AlRahabi MK. Evaluation of complications of root canal treatment performed by undergraduate dental students. Libyan J Med. 2017;12(1):1345582.

6. Ensinas P, Cornejo N, Ramos M L. Apical morphology of lower first molar mesial roots in a Northern argentine population. A scanning electron microscopy stud. RAOA. 2011;99(3):193-200.

7. Greco-Machado Y, García-Molina JA, Lozano-De Luaces V, Manzaranes-Céspedes MC. Morfología de los conductos radiculares de premolares superiores e inferiores. Endodoncia. 2009;27(1):13-18. 8. Duigou $\mathrm{CH}$. Discuss The Prevention And Management of Procedural Errors During Endodontic Treatment. AustrEndod J. 2004;30(2):75-78.

9. Chugal NM, Clive JM, Spångberg LS. Endodontic infection: some biologic and treatment factors associated with outcome. Oral Surg Oral Med Oral Pathol Oral Radiol Endod. 2003;96(1):81-90.

10. Friedman S, Mor C. The success of endodontic therapy-healing and functionality. J Calif Dent Assoc. 2004;32(6):493-503.

11. Imura N, Pinheiro ET, Gomes BP, Zaia AA, Ferraz CCR, Souza-Filho

FJ. The outcome of endodontic treatment: a retrospective study of 2000 cases performed by a specialist. J Endod. 2007;33(11):1278-1282. 12. Sunay H, Tanalp J, Dikbas I, Bayirli G. Cross-sectional evaluation of the periapical status and quality of root canal treatment in a selected population of urban Turkish adults. Int Endod J. 2007;40(2):139-145.

13. Khabbaz M, Protogerou E, Douka E. Radiographic quality of root fillings performed by undergraduate students. Int Endod J. 2010;43(6):499-508.

14. Eleftheriadis G, Lambrianidis T. Technical quality of root canal treatment and detection of iatrogenic errors in an undergraduate dental clinic. Int Endod J. 2005;38(10):725-734.

15. Gonzalez Sanchez JA, Duran-Sindreu F, Albuquerque Matos
M. Apical transportation created using three different patency instruments.Int Endod J. 2010;43(7):560-564.

16. Gonzalez Sanchez JA, Duran-Sindreu F, de Noe S, Mercade M, Roig M. Centring ability and apical transportation after over instrumentation with ProTaper Universal and ProFile Vortex instruments. Int Endod J. 2010;45: 542-551.

17. Chueh LH, Technical quality of root canal treatment in Taiwan. Int Endod J. 2003;36: 416-422

18. Ontiveros Gutiérrez A, Cobos Hernández E, Espinosa Torres N, Espinoza Torres A. Análisis de la transportación en el tercio apical utilizando la Técnica de instrumentación de fuerzas balanceadas vs sistema de instrumentación rotatoria MTWO. Revista ADM. 2012; 69(5):226-232.

19. Lynch C.D; Burk F.M. Quality of root canal fillings performed by undergraduate dental students on single-rooted teeth. Eur J Dent Educ.2006;10:67-72.

20. Alcota Rojas M, Zepeda Zarate C. Calidad técnica de la obturación radiográfica de tratamientos realizados por estudiantes del posgrado en Endodoncia de la Universidad de Chile. Endodoncia.2010;4:215-219.

21. Wolfson E. M., Seltzer S. Reaction of rat connective tissue to some gutta-percha formulations. Journal of Endodontics. 1975;1(12):395-402.

22. Yousuf W, Khan M, Mehdi H. Endodontic Procedural Errors: Frequency, Type of Error, and the Most Frequently Treated Tooth. Int J Dent. 2015;2015:673914

23. Baumann MA, Roth A. Effect of experience on quality of canal preparation with rotary nickel-titanium files,Oral Surg Oral Med Oral Pathol Oral RadiolEndod.2015;88:714 -718.

24. Kerekes K, Tronstad L. Long-term Results of Endodontic Treatment Performed with a Standardized Technique.JEndod. 1979;5(3): 83-90.

25. Barrieshi-Nusair K.M; Al-Omari M.A; Al-Hiyasat A.S. Radiographic technical quality of root canal treatment performed by dental students at the dental teaching center in Jordan. J Dentistry. 2004;32: 301-307.

26. Haug SR, Solfjeld AF, Ranheim LE, Bardsen A. Impact of case difficulty on endodontic mishaps in an undergraduate student clinic. J Endod. 2018;44(7):1088-95.

27. Ribeiro DM, Réus JC, Felippe WT, Pacheco-Pereira C, Dutra KL, Santos JN, Porporatti AL, De Luca Canto G. Technical quality of root canal treatment performed by undergraduate students using hand instrumentation: a metanalysis. Int Endod J. 2018;51(3):269-283.

28. Hendi SS, Karkehabadi H, Eskandarloo A. Iatrogenic Errors during root canal instrumentation performed by dental students. Iran Endond J. 2018;13(1):126-131. 